\title{
Estimación del coste social del consumo de drogas ilegales en Catalunya
}

\section{An estimation of the social cost of illicit drug consumption in Catalonia}

\author{
Vincenzo Vella*, Nuria Ibáñez**, Lidia Segura**, Joan Colom**, Anna García-Altés*,***,****. \\ * Agència de Qualitat i Avaluació Sanitàries de Catalunya (AQuAS), Departament de Salut. \\ Generalitat de Catalunya, Barcelona. España. \\ ** Sub-direcció General Drogodependències, Secretària de Salut Pública de Catalunya, Departament de Salut. \\ Generalitat de Catalunya, Barcelona. España. \\ *** CIBER de Epidemiología y Salud Pública (CIBERESP), Madrid. España. \\ **** Institut d'Investigació Biomèdica (IIB Sant Pau), Barcelona. España.
}

\section{Resumen}

Mundialmente, así como en España, el consumo de drogas ilegales es uno los principales contribuyentes a la carga mundial de morbilidad. Cuantificar los costes que las drogas ilegales imponen a la sociedad es clave para la toma de decisiones. El objetivo de este trabajo es estimar el coste social del consumo de drogas ilegales en Cataluña para un año específico y establecer una metodología para poder replicar dichas estimaciones regularmente y monitorear el impacto de los planes nacionales. Se ha realizado un estudio de coste de la enfermedad. Para la estimación de los costes de mortalidad y morbilidad se ha utilizado el enfoque de la fracción atribuible. Solo se incluyeron los costes del sector público, sanitarios y no sanitarios. El coste del consumo de drogas ilegales en Cataluña en 2011 se estimó en 326,39 millones de € $(0,16 \%$ del PIB catalán en 2011; 0,15\% en 2018). El 82\% del coste total correspondió a costes directos; de estos, el 30,32\% correspondió al sistema penal, $15,99 \%$ a hospitalizaciones, $13,48 \%$ a la policía, $17,19 \%$ a farmacia, $8,34 \%$ a tratamiento en centros especializados y $5,74 \%$ a comunidades terapéuticas, entre otros. Los costes indirectos representaron el $18 \%$ de los costes totales, principalmente pérdidas de productividad debidas a muertes por el consumo de drogas. Este estudio ha sido una oportunidad para recopilar datos de forma sistemática y pensar en los posibles rendimientos económicos que podrían obtenerse de políticas y programas efectivos destinados a reducir el consumo de drogas ilegales. Palabas clave: Drogas ilegales; coste de la enfermedad; costes sociales; política; planes nacionales.

\begin{abstract}
Worldwide, as well as in Spain, the use of illegal drugs is among the major contributors to the global burden of disease. Quantifying the costs that illegal drugs impose on society is key in terms of decisionmaking. The objective of this paper is to estimate the social cost of illicit drug consumption in Catalonia for a specific year, and to establish a methodology to be able to replicate such estimations regularly and monitor properly the impact of national plans. To do that, a cost of illness study was performed. For the estimation of mortality and morbidity costs, we relied on the Attributable Fraction approach. Only public sector costs were included: healthcare and nonhealthcare costs. The cost of illegal drug consumption in Catalonia in 2011 was estimated at $€ 326.39$ million $(0.16 \%$ of the Catalan GDP in $2011 ; 0.15 \%$ in 2018). Of the total cost, $82 \%$ corresponded to direct costs. Among direct costs, $30.32 \%$ corresponded to the penal system, $15.99 \%$ to hospitalizations, $13.48 \%$ to the police force, $17.19 \%$ to pharmacy, $8.34 \%$ to treatment in specialized centres, and $5.74 \%$ to therapeutic communities, among others. Indirect costs represented $18 \%$ of total costs, mostly lost income due to drug-related death. This study has been an opportunity to systematically collect data and think about the potential economic returns that could be achieved from effective policies and programs aimed at reducing the consumption of illegal drugs.

Key Words: Illegal drugs; cost of illness; social cost; policy; national plans.
\end{abstract}




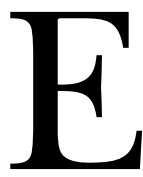

1 consumo de drogas ilegales a nivel mundial es uno los principales factores que contribuyen a la carga mundial de morbilidad. En el año 2015 contribuyeron con la perdida de 111 millones de años de vida ajustados en función de la discapacidad (AVAD). La exposición de riesgo a las drogas a nivel mundial para ambos sexos ha aumentado de manera significativa en los últimos 25 años. Como quinto factor de riesgo para los hombres, los trastornos por uso de sustancias se asociaron con el 6,6\% de la carga de morbilidad; para mujeres, fueron el duodécimo factor de riesgo, con el 2\% (IHME, 2016).

La prevalencia anual global estimada del consumo de drogas ilegales fue más elevada para cannabis $(3,8 \%$ de los adultos con edades entre 15-64 años), seguido de anfetaminas $(0,77 \%)$, opioides (incluso opioides recetados y opiáceos; $0,37 \%)$ y cocaína $(0,35 \%)$ (UNODC, 2017$)$. También se estimó que el $0,25 \%$ de la población adulta con edades entre 15-64 años informó haberse inyectado drogas en el 2015, equivalente a 11,8 millones de personas. La dependencia de cannabis y opioides fueron la dependencia de sustancias ilegales más comunes, con 19,8 y 16,8 millones de casos, respectivamente. La dependencia de anfetaminas y cocaína tuvieron menor prevalencia, con 6.6 millones y 3,9 millones de casos, respectivamente (Peacock et al., 2018). Mundialmente, las tasas de mortalidad estandarizadas por edad fueron 6,9 muertes por 100000 personas en 2015 para sustancias ilegales (Peacock et al., 2018). La carga atribuible a las drogas ilegales se concentró en trastornos por uso de sustancias (16,9 millones), de los cuales 12,9 fueron atribuibles a los trastornos por uso de opioides, cirrosis (4,7 millones), infección por VIH (3 millones) y cáncer hepático (1,8 millones) (IHME, 2016). Los daños que causan las drogas ilegales a los demás -como familias, comunidades y la sociedad en general- también son muchos y amplios, desde la violencia hasta las pérdidas de producción (Nutt, King y Phillips, 2010).

En España, junto con el uso de alcohol, el uso de drogas ilegales ocupa la sexta posición entre los factores de riesgo en relación a la carga de morbilidad (los AVAD) (IHME, 2016). España está entre los países con el consumo más elevado de drogas ilegales (European Monitoring Centre for Drugs and Drug Addiction, 2016). Hasta el 2\% de los españoles reporta el uso diario de cannabis, y el 7\% el uso diario de sedantes (Observatorio Español de la Droga y las Toxicomanías, 2016). El policonsumo de drogas es habitual en el $95 \%$ de las ocasiones junto con el alcohol y en el $60 \%$ junto con el cannabis, donde la vía más frecuente es «tabaco-uso diario de tabaco-cannabis-cocaína» (Sánchez-Niubò, 2020). Cataluña es la región española en particular con la prevalencia más elevada (hasta 50\%) de Hepatitis C, en relación al uso intravenoso de drogas (Saludes et al., 2019). España también es uno de los principales países de Europa en cuanto a drogas decomisadas, especialmente cannabis y heroína (Observatorio Europeo de las Drogas y las Toxicomanías, 2016).

En la actualidad coexisten en Europa varios tipos de governanza en el ámbito de las addicciones. Algunos países introducen estrategias innovadoras para disminuir los efectos negativos; otros aún dependen de enfoques más tradicionales (Anderson et al., 2017). Independientemente del enfoque gubernamental, cuantificar la prevalencia de uso y las cargas de morbilidad y mortalidad asociadas a nivel de país, y acompañar esto de un análisis de los costes directos e indirectos que las drogas ilegales suponen para la sociedad, es clave y debe informar la planificación de políticas y la evaluación de la provisión de servicios (Degenhardt y Hall, 2012).

Sin embargo, para realizar estos estudios, los datos disponibles sobre el gasto público en los costes sociales del abuso de las drogas ilegales en Europa todavía son escasos y heterogéneos, tanto a nivel local como nacional. Varios estudios sugieren que estos costes son elevados, pero aún hay mucha controversia y una falta de consenso sobre cómo calcular los costes de este fenómeno complejo (Barrio, Reynolds y García-Altés, 2017). Una revisión reciente realizada en el contexto del proyecto LEADER ha generado una propuesta de dos marcos (mínimo e ideal) para guiar la estimación de los costes sociales en investigaciones futuras (Vella, García-Altés, Segura García, Ibáñez Martínez y Colom Farran, 2018), junto con un documento guía sobre los métodos para calcular la estimación (LEADER, 2017). Contar con un enfoque estándar de cálculo en el ámbito social también puede facilitar en análisis del impacto económico de implementar las estrategias nacionales sobre drogas, como hizo recientemente Portugal, donde los costes sociales de uso de drogas disminuyó $18 \%$ en el período de once años tras la aprobación de la estrategia portuguesa para la lucha contras las drogas (Gonçalves, Lourenço y Nogueira da Silva, 2015).

El gasto relacionado con el uso de drogas entre los 18 países que han publicado sus estimaciones en los últimos 10 años se calcula entre $0,01-0,5 \%$ del PIB, representando las intervenciones relacionadas con la salud y con diferencias importantes entre los países (Observatorio Europeo de las Drogas y las Toxicomanías, 2016).

En España, se han implementado esfuerzos significativos en décadas recientes para contrarrestar los efectos del consumo de drogas a nivel individual y social en general. En este sentido, se han implementado, coordinado y hecho un seguimiento de diferentes planes a nivel nacional y regional. Los esfuerzos incluyeron intentos para estimar el coste social del abuso de drogas ilegales (García-Altés, Ollé, Antoñanzas y Colom, 2002; Rivera, Casal y Currais, 2017). Los autores estimaron que en 1997 el coste mínimo del consumo de drogas ilegales en España alcanzó el 0,07\% del PIB, mientras que para Galicia representó el 0,12\% del PIB en 2008 (Portella et al., 2003). 
Como parte de un esfuerzo por mejorar las herramientas de planificación en el contexto de la evaluación del plan catalán sobre drogas, este estudio tiene como objetivo estimar el coste social del uso de drogas ilegales en Cataluña en 2011.

\section{Métodos}

El sistema de salud de Catalunya (una región de España) está organizada como un sistema nacional de salud financiado con impuestos. Todos los residentes (7.348.275 en 2017) tienen derecho legal a la atención sanitaria universal. El gasto público para la atención sanitaria representa el 5,4\% del PIB catalán.

Realizamos un estudio de costes de la enfermedad (COI), que ha sido ampliamente implementado para calcular los costes sociales del abuso de sustancias (García-Altés et al., 2002; Godgrey, Eaton, McDougall y Culyer, 2002; Kopp, 2001; Rehm et al., 2006; Rivera et al., 2017). Como con cualquier estudio de costes de la enfermedad, la contrapartida es que los costes se calculan en comparación con una alternativa hipotética de ausencia de la condición de interés, en nuestro caso la no existencia del uso de drogas ilegales.

Para la estimación de los costes de mortalidad y morbilidad se ha utilizado el enfoque de la fracción atribuible (FA) (Rehm et al., 2006). La FA estima la proporción de la enfermedad entre los expuestos a un factor de riesgo específico, en nuestro caso el uso de drogas ilegales. Las FA se calculan según la siguiente fórmula (Rehm et al., 2006):

$$
A F=\frac{\left[\sum_{i=1}^{k} P_{i}\left(R R_{i}-1\right)\right]}{\left[\sum_{i=1}^{k} P_{i}\left(R R_{i}-1\right)+1\right]}
$$

Donde:

- $i$ es la categoría de exposición. La línea basal (la no exposición) es $\mathrm{i}=0$;

- $\operatorname{RR}(i)$ indica el riesgo relativo al nivel de exposición $i$ comparado con el no consumo de drogas.
- $\mathrm{P}(i)$ indica la prevalencia de la categoría de exposición a la enésima potencia de $i$.

Incluimos los costes directos e indirectos de la salud pública y excluimos los costes privados y los intangibles (Kopp, 2001). La matriz adoptada para el coste de referencia es comparable con dos de los estudios más recientes realizados en países europeos (Gonçalves et al., 2015; Rivera et al., 2017). Estas contribuciones son coherentes con las referencias clásicas (Kopp, 2001; Single et al., 2003) en cuanto a la definición de los costes sociales y la categorización de los ítems. La Tabla 1 muestra las categorías estimadas.

Todos los datos públicamente disponibles se obtuvieron de entidades de los sistemas catalanes de salud pública y justicia. Identificamos en el artículo la fuente de cada dato al presentarlo, además del supuesto adoptado para calcularlo.

\section{Costes directos relacionados con la salud}

Los costes de los tratamientos se desglosaron de la siguiente manera: farmacia, incluso todos los costes de medicamentos recetados para tratar las consecuencias del uso de drogas ilegales, visitas a centros de atención primaria, tratamiento en centros especializados y tratamiento de adicciones en centros penitenciarios (Gerencia de Atención Farmacéutica y Prestaciones Complementarias). Identificamos cinco medicamentos disponibles con receta como relevantes en el tratamiento de usuarios de drogas ilegales: metadona (8.055 personas en terapia de sustitución de opiáceos), buprenorfina (4.189 personas), naltrexona (2.263 personas), benzodiazepinas, y terapia antirretroviral para VIH (4.484 personas). Los primeros tres se consideraron $100 \%$ dedicados al tratamiento de usuarios de drogas ilegales. Para las benzodiazepinas, solo el $1 \%$ para hombres y $0,5 \%$ para mujeres fue atribuible al tratamiento de usuarios de drogas. Esto se aplicó a la población de usuarios con edades entre 18-65 años para excluir los casos de consumo no relacionado con drogas ilegales. Para estimar el coste del tratamiento antirretroviral para VIH, identificamos el coste total de 2011 para estos medicamentos en Cataluña, sumando a 152.092.275€. Aplicamos a este presupuesto la FA correspondiente (Rehm

Tabla 1. Matriz de costes.

\begin{tabular}{lll}
\hline Tipo de coste & Coste directo & Coste indirecto \\
\hline Relacionados con la salud & Costes de tratamientos & $\begin{array}{l}\text { Pérdida de ingresos debido a fallecimiento a causa del uso } \\
\text { de drogas }\end{array}$ \\
\cline { 2 - 3 } & Hospitalización & $\begin{array}{l}\text { Pérdida de productividad debido a tratamiento y/o } \\
\text { hospitalización }\end{array}$ \\
\cline { 2 - 2 } & Investigación y prevención \\
\cline { 2 - 2 } & Programas de apoyo contra la adicción & \\
\hline No relacionados con la salud & Justicia & \\
\cline { 2 - 2 } & Policía & Sistema penal \\
\cline { 2 - 2 }
\end{tabular}


et al., 2006) (21\%) para obtener la parte del presupuesto aplicable al tratamiento de personas usuarias de drogas ilegales $(32.851 .931,40 €)$. Aunque se calcula que entre el 60$80 \%$ de los usuarios de drogas están infectados con hepatitis C, excluimos los costes de los antirretrovirales porque en 2011 muy pocas personas con adicción fueron tratadas debido a los efectos adversos y baja efectividad del tratamiento con interferones y ribavirina.

Identificamos las visitas a centros de atención primaria relacionadas con el uso de drogas ilegales según el Conjunto Mínimo Básico de Datos (Servicio Catalán de Salud) y los códigos de la CIE (versión 10) de adicción a cocaína y opioides. Así, fue posible identificar el número de visitas a médicos, enfermeras y servicios sociales y aplicar el coste de cada tipo de visita (Vela, Clèries, Vella, Adroher y García-Altés, 2019).

Para calcular los costes de los tratamientos en centros especializados, aplicamos la proporción de pacientes que acudió a estas consultas por adicción a drogas $(49,4 \%$ del total) al presupuesto total invertido en esos servicios (CatSalut, 2012) (20.510.803,39€). Después añadimos los costes de las unidades de desintoxicación $(1.746 .359,13 €)$.

Estimamos el coste de los tratamientos para adicción a drogas ilegales según los datos aportados por el Departamento de Salud de Cataluña y otras organizaciones no gubernamentales. El coste de tratar a 3980 presos con adicción en las prisiones catalanas sumó a 5.062.116,66€.

Repartimos los costes de hospitalización en las siguientes subcategorías: costes de morbilidad para tratamientos de pacientes ingresados; costes de emergencias y costes de servicios de ambulancia.

Los registros de pacientes hospitalizados por consumo de drogas ilegales se obtuvieron de la base de datos de altas hospitalarias (Registro de Actividad de Atención Especializada. RAE-CMBD). Multiplicamos cada coste relacionado con el GRD por su FA correspondiente (Rehm et al., 2006).

La base calcular «emergencias» fue los datos de visitas a los servicios de urgencias aportados por tres hospitales en Barcelona. Calculamos el número promedio de visitas a urgencias de estos hospitales, considerando su población de referencia total. Entonces aplicamos el resultado de este cálculo a la población total de Cataluña en 2011. Calculamos el coste unitario para las visitas a urgencias como la media ponderada entre la tarifa de reembolso de dos categorías de hospitales, general y especializado, utilizando como pesos las frecuencias relativas de uso en 2011.

Obtuvimos del Sistema de Emergencias Médicas de Cataluña el número de servicios de ambulancias específicos para emergencias relacionadas con el uso de drogas ilegales, y aplicamos un coste unitario a través de una media ponderada del precio del servicio básico y una ambulancia de soporte vital avanzado (Orden de 17/11/2014, 2014).

Calculamos los gastos de investigación relacionados con el consumo de drogas ilegales como la suma del total de los fondos de las tres convocatorias principales para estudios en este ámbito: el Plan Nacional sobre Drogas (2011), la Red de Trastornos Adictivos (RETOX) y la Agencia de Gestión de Ayudas Universitarias y de Investigación (AGAUR).

El cálculo de los gastos en prevención tuvo en cuenta el presupuesto del Departamento de Salud de Cataluña además de además de los fondos específicos adjudicados por el Plan Nacional sobre Drogas del Gobierno de España. Incluimos solo aquellos fondos destinados específicamente para Cataluña y su población o la parte de los programas nacionales centrados en Cataluña.

Respecto de los costes de rehabilitación e inclusión social, incluimos los costes asociados al número de personas beneficiarias de servicios de comunidades terapéuticas y de rehabilitación (Departamento de Salud de Cataluña y Departamento de Bienestar Social y Familia de Cataluña). También incluimos el coste del teléfono de información sobre drogodependencias «Línea Verda» según el informe proporcionado por el servicio mismo.

\section{Costes directos no relacionados con la salud}

Para los costes de justicia, obtuvimos el número de procedimientos judiciales relacionados con el tráfico de drogas en Cataluña para 2011 (Observatorio Español de la Droga y las Toxicomanías, 2013) y aplicamos un coste estimado de $415 €$ por procedimiento (Rivera et al., 2017).

El cuerpo policial autonómico de Cataluña proporcionó los datos sobre costes policiales de los controles de conducción bajo la influencia de drogas, policía científica y seguridad pública; el Plan Nacional sobre Drogas aportó datos sobre el control del tráfico de drogas, y la Dirección General de Tráfico sobre pruebas policiales de uso de drogas entre conductores de vehículos implicados, o no, en accidentes de tráfico.

Aplicamos un coste medio de 20.340€ para el sistema penal, correspondiente al coste de manutención de los 3980 personas recluidas (Rivera et al., 2017) con adicción en los centros penitenciarios catalanes.

\section{Costes indirectos relacionados con la salud}

Para el cálculo de las pérdidas en productividad, aplicamos la FA para estimar los costes generados por las muertes relacionadas con el consumo de drogas ilegales (Rehm et al., 2006). Identificamos los ingresos anuales potenciales según el salario promedio anual per cápita para 2011 en Cataluña (presuntamente igual para hombres y mujeres) (Idescat, 2017). Actualizamos el ingreso anual futuro utilizando un enfoque típico de valor presente neto, utilizando una tasa de descuento del 3\%. La fórmula utilizada para calcular la pérdida de ingresos (lost income) fue:

$$
\text { Lost income }=N_{t}^{D R D} \cdot\left[\frac{R_{t}\left(1-(1+r)^{-m_{t}}\right.}{r}\right]
$$


Donde $N_{t}^{D R D}$ es el número de muertes relacionadas con drogas ilegales; representa el salario promedio anual; $R_{t}$ es la diferencia en años entre la edad de jubilación (65 años para hombres y mujeres) y la edad en el momento de la muerte. En otras palabras, la pérdida de ingresos es el valor actualizado del salario no cobrado a causa del fallecimiento. Agrupamos a los individuos en grupos por edad para calcular los fallecimientos. Esto era coherente con las contribuciones principales en la literatura (Rehm et al., 2006). Solo tuvimos en cuenta a las personas aún en edad laboral, por lo que excluimos los fallecimientos en los grupos de 70-79 años y $>80+$ años.

Estimamos las pérdidas de ingresos con un enfoque basado en el método de valor añadido bruto (VAB) neto de salarios (Gonçalves et al., 2015). Aplicamos el VAB diario (Idescat, 2018) al número de días de hospitalización asociado a los datos de morbilidad utilizados para estimar los costes de hospitalización (véase las secciones anteriores). Respecto de las pérdidas de productividad, aplicamos la FA a los datos de días de hospitalización. La estimación del VAB diario fue $95,93 €$.

\section{Resultados}

Como muestra la Tabla 2, el coste del consumo de drogas ilegales en Cataluña para 2011 se estimó en 326,39€ millones. El $82 \%$ del coste total correspondió a costes di- rectos, distribuidos de manera muy equitativa entre gastos relacionados y no relacionados con la salud. De los costes directos, 44,37\% correspondió a los costes no relacionados con la salud (justicia, policía y sistema penal), 15,99\% a hospitalizaciones, $17,19 \%$ a farmacia, $8,34 \%$ a tratamiento en centros especializados, y $5,74 \%$ a comunidades terapéuticas y otros programas asistenciales, entre otros. Los costes indirectos representaron el $18 \%$ de los costes totales, la mayoría por pérdida de ingresos debido a fallecimiento a causa del uso de drogas (Figura 1).

\section{Costes directos relacionados con la salud}

Los costes farmacéuticos se estimaron en 45.886.505,83€. De estos, la mayor parte fue por los tratamientos del VIH $(71,59 \%)$. Los tratamientos en centros especializados, incluídas unidades de desintoxicación y centros de tratamiento de drogodependencias para pacientes ambulatorios, suman a 22.257.162,52€; visitas a centros de atención primaria, 3.515.452€ y tratamiento en instituciones penitenciarias, 5.062.116,66€ (Tabla I, anexo en línea).

Identificamos un total de 8.385 casos de hospitalización relacionados con el uso de drogas ilegales, lo que representó 42.696.460,35€ en costes hospitalarios. Los pacientes masculinos generaron el $86,56 \%$ de los costes totales, de los cuales VIH y hepatitis C representaron $16 \%$ y $24 \%$, respectivamente. Las enfermedades que generaron un uso más elevado de los recursos para mujeres fueron complica-

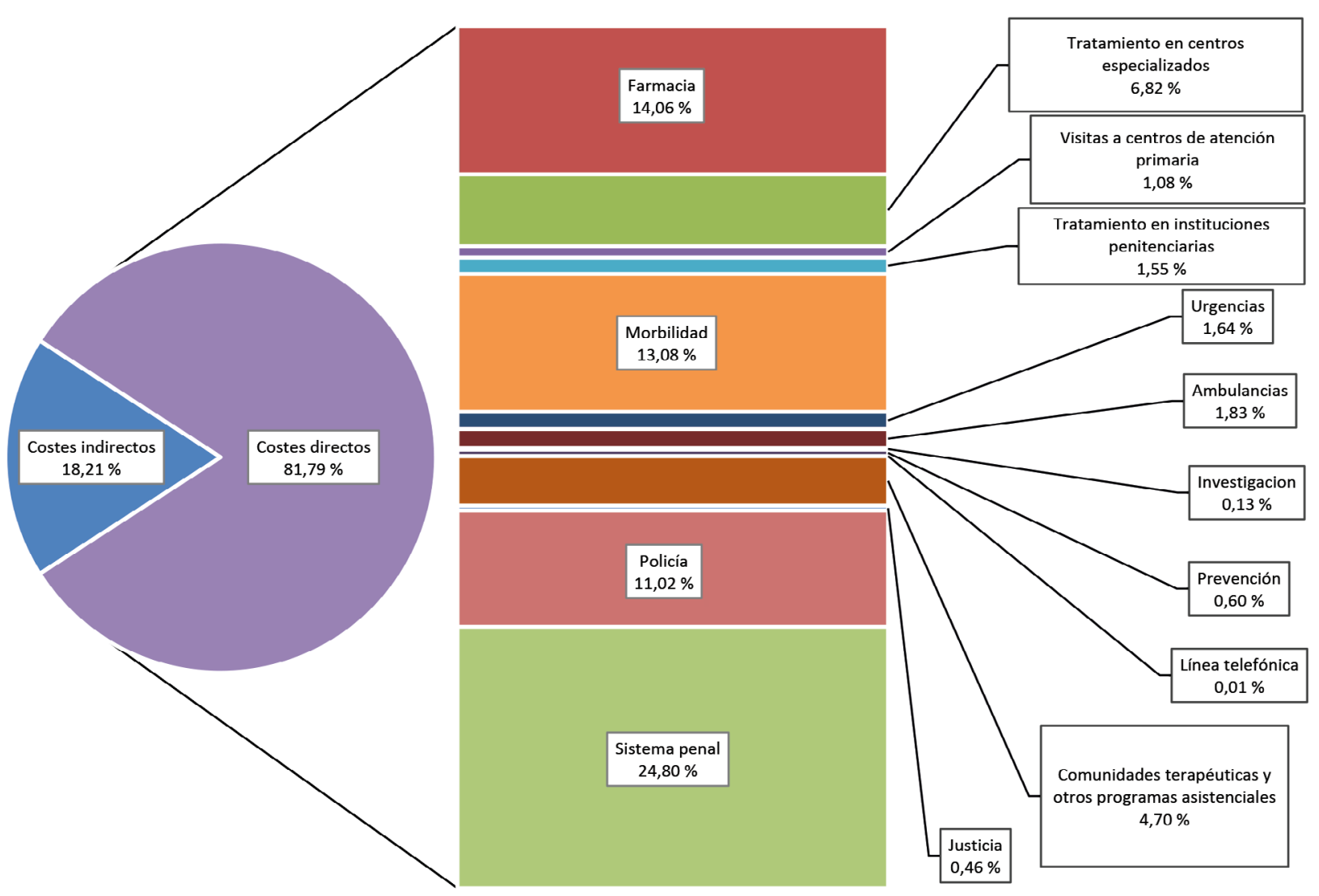

Figura 1. Distribución de costes directos e indirectos. Cataluña, 2011. 
Tabla 2. Coste de consumo de drogas ilegales por tipo de coste. Cataluña, 2011.

\begin{tabular}{|c|c|c|c|}
\hline Tipo de coste & & Ítem & Cuantía \\
\hline \multicolumn{4}{|l|}{ Costes directos } \\
\hline \multirow[t]{11}{*}{ Relacionados con la salud } & Costes de tratamientos & Farmacia & $45.886 .505,83 €$ \\
\hline & & Tratamiento en centros especializados & $22.257 .162,52 €$ \\
\hline & & Visitas a centros de atención primaria & $3.515 .452,00 €$ \\
\hline & & Tratamiento en instituciones penitenciarias & $5.062 .116,66 €$ \\
\hline & Hospitalización & Morbilidad & $42.696 .460,35 €$ \\
\hline & & Urgencias & $5.352 .194,97 €$ \\
\hline & & Ambulancias & $5.968 .429,79 €$ \\
\hline & Investigación y prevención & Investigación & $436.568,75 v$ \\
\hline & & Prevención & $1.952 .888,55 €$ \\
\hline & Programas de apoyo contra la adicción & $\begin{array}{l}\text { Teléfono de información sobre } \\
\text { drogodependencias }\end{array}$ & $45.399,84 €$ \\
\hline & & $\begin{array}{l}\text { Comunidades terapéuticas y otros programas de } \\
\text { rehabilitación e inclusión social similares }\end{array}$ & $15.332 .085,96 €$ \\
\hline \multirow[t]{3}{*}{ No relacionados con la salud } & Justicia & & $1.509 .355,00 €$ \\
\hline & Policía & & $35.977 .463,81 €$ \\
\hline & Sistema penal & & $80.953 .200,00 €$ \\
\hline \multicolumn{4}{|l|}{ Costes indirectos } \\
\hline \multirow[t]{2}{*}{ Relacionados con la salud } & $\begin{array}{l}\text { Pérdida de ingresos debido a fallecimiento a } \\
\text { causa del uso de drogas }\end{array}$ & & $57.405 .516,65 €$ \\
\hline & $\begin{array}{l}\text { Pérdida de productividad debido a } \\
\text { tratamiento y/o hospitalización }\end{array}$ & & $2.039 .622,66 €$ \\
\hline \multirow[t]{2}{*}{ Total costes directos } & Relacionados con la salud & & $148.505 .265,22 €$ \\
\hline & No relacionados con la salud & & $118.440 .018,81 €$ \\
\hline Total & & & $266.945 .284,03 €$ \\
\hline Total costes indirectos & & & $59.445 .139,30 €$ \\
\hline Total costes sociales & & & $326.390 .423,33 €$ \\
\hline
\end{tabular}

ciones durante el parto y hepatitis C, $37 \%$ y $25 \%$, respectivamente. La contribución de cada enfermedad figura en la Tabla II, anexo en línea.

Las visitas a urgencias representaron 5.352.194,97€, generado de 31858 visitas asociadas al uso de drogas ilegales. El total de casos de servicios de ambulancia se estimó en 12283 (hombres: 54\%; mujeres: 44\%; sexo no informado: $2 \%$ ), con un coste total de 5.968.429,79€.

La investigación representó el 0,36\% de los costes directos. La contribución de cada centro de investigación figura en la Tabla III, anexo en línea.

Los costes de prevención alcanzaron los 1.952.888,55€. Los programas de apoyo contra la adicción, de rehabilitación y de inclusión social sumaron a 15.332.085,96€. El coste del teléfono de información sobre drogodependencias fue de $45399.84 €$.

\section{Costes directos no relacionados con la salud}

En 2011, se identificaron 3.980 juicios en relación a las drogas ilegales, representando un coste de 1.509.355€ (0,56\% de los costes directos).
Los cuerpos de seguridad están implicado en la lucha contra el consumo y el tráfico de las drogas ilegales. Las actividades más relevantes se reflejan en la Tabla IV anexo en línea, que estima la contribución de cada componente y suma a un total de $35.977 .463,81 €$, entre los cuales las actividades de control de tráfico de drogas y el Centro de Inteligencia Contra el Crimen Organizado (CICO) representaron el 41,64\% del mismo. Un total de 3980 presos estaban encarcelados por delitos relacionados con las drogas ilegales, lo que generó un total de 80.953.200€ (30,32\% de los costes directos).

\section{Costes indirectos relacionados con la salud}

Hubo un total de 151,85 muertes asociadas con las drogas ilegales en 2011, lo que representó una pérdida de ingresos futuros de 57.405.516,65€ (94,45\% de los costes indirectos) (Table $\mathrm{V}$ anexo en línea). Los hombres representaron el 71,20\% del total. El VIH fue la enfermedad que provocó el mayor número de fallecimientos (especificado como B20-B24 en la tabla), seguido de suicidio (X60-X84, Y87.0). 
Respecto de las pérdidas de productividad, el total de 21274.11 días laborales perdidos generó un total de costes de 2039 622.66€ (Table VI anexo en línea).

\section{Discusión}

Este estudió calculó que el coste social mínimo del consumo de drogas ilegales en Cataluña en 2011 fue 326,39 millones de $€(349,56$ millones de $€$ en 2018). Para poner dichas cifras en contexto, el coste social del consumo de drogas ilegales representó el $0,16 \%$ del PIB catalán en 2011 (0,15\% en 2018). Además, esta cifra ha de compararse con la inversión de 1,27 millones de € en programas de prevención el mismo año. Estimaciones anteriores para España: 1,436 billones de euros en 2012 (0,14\% del PIB de España) (Rivera et al., 2017) y 88,8 billones de pesetas en 1997 (aproximadamente 836,30 millones de $€$ en la actualidad) (García-Altés et al., 2002).

Adoptamos el marco del COI, ampliamente implementado para calcular los costes sociales del abuso de sustancias (Godgrey et al., 2002; Kopp, 2001; Rehm et al., 2006), también para los ejemplos de España (García-Altés et al., 2002; Rivera et al., 2017). Las ventajas y las desventajas del COI se han analizado en profundidad tanto desde una perspectiva general como en relación a nuestro campo de interés (Collins y Lapsley, 2002; Single et al., 2003). Esto ha fomentado el desarrollo de una variedad de enfoques alternativos y soluciones específicas a los límites del COI cuando se aplica al caso de las drogas ilegales (Portella et al., 2003; Rehm et al., 2006; Single et al., 2003). Además, también se han sugerido métodos alternativos para calcular los costes indirectos (Koopmanschap, Rutten, van Ineveld y van Roijen, 1995). El marco adoptado por este estudio depende del COI y también tiene en cuenta mejoras posteriores (Gonçalves et al., 2015; Kopp, 2001; Rehm et al., 2006).

No obstante, las estimaciones generadas en este estudio son más altas que las de otros. Nuestra hipótesis es que la estimación más alta se debe 1) al enfoque innovador utilizado y el esfuerzo por incluir la mayor parte posible de los datos disponibles a nivel local; 2) la inclusión de la mayor parte de las categorías de Rehm et al. (2006) en el marco, incluso las que tienen mayor impacto en los costes sociales, como pérdidas de productividad; 3) la inclusión de todas las categorías de enfermedad en el análisis de los costes de mortalidad y morbilidad.

Uno de los problemas principales al estimar el coste social del uso de drogas durante un período específico es la definición del análisis contrafactual. La opción más común en la literatura es suponer que la ausencia de uso de sustancias en el pasado, presente y futuro como contrafactual (Gonçalves et al., 2015; Kopp, 2001; Rivera et al., 2017). Aunque adoptado con frecuencia, este enfoque ha sido objeto de críticas (Collins et al., 2002; Single et al., 2003). Los críticos defienden considerar solo parte de los costes totales en la estimación, i.e., la que es susceptible de las políticas públicas; se definen como costes evitables. En su lugar, los costes totales incluyen gastos que no pueden ser afectados por las políticas actuales; por ejemplo, los relacionados con el uso de sustancias en el pasado o los generados por casos de uso de sustancias en el pasado que continuará en el futuro (Collins et al., 2002; Single et al., 2003). Al adoptar el caso de un consumo cero como contrafactual, estos costes se incluyen de forma automática en la estimación de los costes sociales. De ahí surge la crítica a este enfoque. Por tanto, deben considerarse como inevitables y no incluidos en el cálculo de los costes sociales (Single et al., 2003). A pesar de estas críticas, no hay otra alternativa en la actualidad que permita calcular estos costes inevitables con precisión (Collins et al., 2002). El enfoque adoptado para este artículo depende del caso de un consumo cero como contrafactual.

La matriz del coste de referencia adoptado es comparable a los anteriores (Gonçalves et al., 2015; Rivera et al., 2017), que representaron dos de los estudios más recientes en países europeos, coherentes con los textos clásicos en cuanto a la definición del coste social y la categorización de los ítems (Kopp, 2001; Single et al., 2003). Porque faltaban algunos datos de los ítems de la matriz del coste, solo pudimos estimar un subconjunto de las categorías incluidas en la matriz de referencia. Es más, no estimamos los costes intangibles debido a las limitaciones inherentes a las metodologías disponibles. No pudimos incluir en los costes específicos los costes de prevención de las administraciones locales y los costes de inclusión social pagados por el Departamento de Trabajo. De forma similar, basamos algunas de las estimaciones en medias y extrapolaciones, como es el caso de las visitas a urgencias, el salario promedio para ambos sexos y los procedimientos judiciales.

Utilizamos datos públicos y privados -principalmente de organizaciones sin ánimo de lucro- para calcular las estimaciones. Hay dos motivos principales por decidir utilizar ambos tipos de fuentes. Primero, nos permitió beneficiarnos de la amplia red de contactos que tienen los autores en el sistema institucional catalán que proporciona distintos tipos de servicios relacionados con el consumo de sustancias ilegales. Esto incluye, entre otros, los costes de las drogas utilizadas para tratamientos de adicción, los programas de investigación científica y los programas de apoyo (como el teléfono de información sobre drogodependencias) para personas con adicción. Segundo, presentar datos de entidades que no suelen incluirse en los cálculos oficiales del coste social del uso de drogas ilegales permitió a estas entidades emerger como red de proveedores de servicios. De todas formas, este estudio es fácilmente replicable, por lo que es sencillo calcular nuevamente las estimaciones, lo que permite hacer un seguimiento adecuado del impacto de los planes nacionales. A la vez, ha 
sido de gran valor establecer relaciones con otros departamentos gubernamentales y aumentar la concienciación sobre el problema de adicción a las drogas, un problema de salud pública donde la «salud en todas las políticas» es completamente relevante.

Ya han pasado 8 años desde 2011, cuando recopilamos estos costes relacionados con las drogas. Es destacable que en 2012, el presupuesto para prevención sufrió un recorte dramático de aproximadamente 1 millón de $€$ debido a la severa crisis económica, y dicha cifra quedó igualada en el 2014 y mantenida sin cambios desde entonces. Una breve revisión de los cambios acontecidos entre el 2011 y los datos disponibles más recientes para la mayoría de los indicadores nos dicen que, a pesar de la ligera fluctuación en los datos, los costes relacionados con las drogas pueden no haber aumentado de forma significativa, con la excepción de los costes para tratamientos farmacológicos. La llegada de nuevos antirretrovirales de acción directa en 2015 ha resultado en un aumento significativo del número de personas que se inyectan drogas (PID) en tratamiento por hepatitis C. Dado que el tratamiento tiene un coste medio de $6.500 €$ por persona y que al menos el $50 \%$ de las PID han estado en tratamiento (7482 personas en 2017), el aumento suma a aproximadamente $24.316 .500 €(7,7 \%$ de los costes relacionados con las drogas en 2011).

En la actualidad coexisten en Europa varios tipos de governanza en el ámbito de las addicciones. Algunos países introducen estrategias innovadoras para disminuir los efectos negativos; otros aún dependen de enfoques más tradicionales (Anderson et al., 2017). Independientemente del enfoque de la administración pública, cuantificar la prevalencia del uso y la carga asociada a la enfermedad y mortalidad a nivel de país, junto con un análisis de los costes económicos directos e indirectos de las drogas ilegales para la sociedad, es clave y debe informar la planificación de políticas y la evaluación de la provisión de servicios (Degenhardt et al., 2012).

\section{Reconocimientos}

Xavier Jiménez Fàbrega, Sistema de Emergencias Médicas, Empresa Pública de la Generalitat de Cataluña.

Sistema de información sobre drogas de Barcelona (SIDB). Agencia de Salud Pública de Barcelona. Consorcio Sanitario de Barcelona.

David Magem y Pere Carbonell, Gerencia de Atención Farmacéutica y Prestaciones Complementarias. Área Sanitaria. Servicio Catalán de Salud (CatSalut).

Programa de abuso de sustancias. Agencia de Salud Pública de Barcelona. Departamento de Salud. Generalitat de Cataluña.

Úrsula Salvador. Línea verda. Servicio de Atención y Orientación Telefónica sobre drogodependencias. Fundación IPSS.
Carme Iniesta i Torres. Responsable de Programas para la Autonomía y la Inserción Social. Subdirección General de Análisis y Programación. ICASS. Departamento de Bienestar Social y Familia.

Albert Batlle i Bastardas. Director general de la policía. Gobierno de Cataluña.

Albert Moya i Salazar, Sargento jefe de la Unidad de Análisis y Planificación. Área Técnica de Coordinación y Soporte División Técnica de Planificación de la Seguridad, Comisaría General Técnica de Planificación de la Seguridad Prefectura de la Policía.

Servicio de Gestión de Asuntos Generales. Centro de Inteligencia contra el Crimen Organizado.

Maria D. Cobos Ruiz. Mercedes Sánchez y Rosa Anna Castillo Picas Subdirección General de Planificación y Programas, Departamento de Justicia.

Xavier Almirall García Responsable de la Oficina del Plan catalán de Seguridad Vial. Servicio Catalán de Tráfico.

Constança Albertí Casas; Neus Rams y Lidia Domingo Ferrer. Instituto catalán de Evaluaciones Médicas. Departamento de Salud. Gobierno de Cataluña.

Glòria Ribas Serra. Responsable de Procesamiento de la Base de Datos de Mortalidad. Servicios de Estudios, Departamento de Salud. Generalitat de Cataluña.

\section{Conflicto de intereses}

Este estudio no recibió ayuda alguna de organismos de financiación de los sectores públicos, privados o sin ánimo de lucro. Los autores declaran la inexistencia de conflicto de interés.

\section{Referencias}

Anderson, P., Berridge, V., Conrod, P., Dudley, R., Hellman, M., Lachenmeier, D.,... Gual, A. (2017). Reframing the science and policy of nicotine, illegal drugs and alcohol - conclusions of the ALICE RAP Project. F1000Research, 6, 289. doi:10.12688/f1000research.10860.1.

Barrio, P., Reynolds, J. y García-Altés, A. (2017). Social costs of illegal drugs, alcohol and tobacco in the European Union: A systematic review of existing publications. Drug and Alcohol Review, 36, 578-588. doi:10.1111/dar.12504.

CatSalut. (2012). Memòria CatSalut. El sistema de salut i la xarxa sanitària pública de Catalunya 2011. Barcelona, Espanya: Departament de Salut. Generalitat de Catalunya. Recuperado de https://catsalut.gencat.cat/ca/ coneix-catsalut/presentacio/informes-memories-activitat/anual-catsalut/.

Collins, D. J. y Lapsley, H. M. (2002). Counting the cost: Estimates of the social costs of drug abuse in Australia in 1998-9. Canberra, Australia: Commonwealth Department of Health and Ageing. Commonwealth of Austra- 
lia. Recuperado de https://catalogue.nla.gov.au/Record/814639.

Degenhardt, L. y Hall, W. (2012). Extent of illicit drug use and dependence, and their contribution to the global burden of disease. Lancet, 379, 55-70. doi:10.1016/ S0140-6736(11)61138-0.

European Monitoring Centre for Drugs and Drug Addiction. (2016). European Drug Report 2016: Trends and Developments. Luxembourg: Publications Office of the European Union. Recuperado de http://www. emcdda.europa.eu/publications/edr/trends-developments/2016_en.

García-Altés, A., Ollé, J. M., Antoñanzas, F. y Colom, J. (2002). The social costs of illegal drugs in Spain. Addiction, 9, 1145-1153. doi:10.1046/j.1360-0443.2002.00170.x.

Godgrey, C., Eaton, G., McDougall, C. y Culyer, A. (2002). The economic and social costs of Class A drug use in England and Wales, 2000. London, United Kingdom: Home Office Research, Development and Statistics Directorate. Recuperado de https://www.drugsandalcohol.ie/5441/1/Home_Office_Research_Study_249_ Economic_and_social_costs.pdf.

Gonçalves, R., Lourenço, A. y Nogueira da Silva, S. (2015). A social cost perspective in the wake of the Portuguese strategy for the fight against drugs. The International Journal on Drug Policy, 26, 199-209. doi:10.1016/j.drugpo.2014.08.017.

Idescat. (2017). Enquesta anual d'estructura salarial. Barcelona, Espanya: Institut d'Estadística de Catalunya (Idescat). Generalitat de Catalunya. Recuperado de https://www.idescat.cat.

Idescat. (2018). Valor afegit brut. Barcelona, Espanya: Institut d'Estadística de Catalunya (Idescat). Generalitat de Catalunya. Recuperado de https:/ / www.idescat.cat.

IHME (2016). GBD Results Tool. Seattle, WA: Institute for Health Metrics and Evaluation (IHME). University of Washington. Recuperado de http://ghdx.healthdata. org/gbd-results-tool.

Kopp, P. (2001). Calculating the social cost of illicit drugs. Methods and tools for estimating the social cost of the use of psychotropic substances. Strasbourg, France: Council of Europe Publishing. Recuperado de https:/ / book.coe.int/en/root/2147-calculating-the-social-costof-illicit-drugs-methods-and-tools-for-estimating-the-social-cost-of-the-use-of-psychotropic-substances.html.

Koopmanschap, M. A., Rutten, F. F., van Ineveld, B. M. y van Roijen, L. (1995). The friction cost method for measuring indirect costs of disease. Journal of Health Economics, 14, 171-89. doi:10.1016/0167-6296(94)00044-5.

LEADER (2017). Looking at Economic Analysis of Drugs and Economic Recession. LEADER European project. Drug Prevention and information (2007-2013). Brussels, Belgium: European Commission. Recuperado de https://www.alicerap.eu/home-leader.html.
Nutt, D. J., King, L. A. y Phillips, L. D. (2010). Drug harms in the UK: A multicriteria decision analysis. Lancet, 376, 1558-1565. doi:10.1016/S0140-6736(10)61462-6.

Observatorio Español de la Droga y las Toxicomanías. (2013). Alcohol, tabaco y drogas ilegales en España. Informe 2013. Madrid, España: Ministerio de Sanidad, Servicios Sociales e Igualdad. Recuperado de https:// www.mscbs.gob.es.

Observatorio Español de la Droga y las Toxicomanías. (2016). Alcohol, tabaco y drogas ilegales en España. Informe 2016. Madrid, España: Ministerio de Sanidad, Servicios Sociales e Igualdad. Recuperado de https:// www.mscbs.gob.es.

Orden de 17/11/2014, de la Consejería de Sanidad y Asuntos Sociales, por la que se establecen los precios públicos de la asistencia sanitaria y de los servicios prestados en la red de centros sanitarios dependientes del Servicio de Salud de Castilla-La Mancha (2014). Toledo, España: Diario Oficial de Castilla-La Mancha (DOCM). Recuperado de https://docm.castillalamancha.es/portaldocm/.

Peacock, A., Leung, J., Larney, S., Colledge, S., Hickman, M., Rehm, J.,... Degenhardt, L. (2018). Global statistics on alcohol, tobacco and illicit drug use: 2017 status report. Addiction, 113, 1905-1926. doi:10.1111/add.14234.

Portella, G., Laezza, C., Laccetti, P., De Petrocellis, L., Di Marzo, V. y Bifulco, M. (2003). Inhibitory effects of cannabinoid CB1 receptor stimulation on tumor growth and metastatic spreading: Actions on signals involved in angiogenesis and metastasis. FASEB J, 17, 1771-1773. doi:10.1096/fj.02-1129fje.

Rehm, J., Baliunas, D., Brochu, S., Fischer, B., Gnam, W., Patra, J.,... Taylor, B. (2006). The costs of substance abuse in Canada 2002. Ottawa, Canada: Canadian Centre on Substance Abuse. Recuperado de https://www.ccsa. ca/sites/default/files/2019-05/ccsa-011332-2006.pdf.

Rivera, B., Casal, B. y Currais, L. (2017). The social cost of illicit drugs use in Spain. The International Journal on Drug Policy, 44, 92-104. doi:10.1016/j.drugpo.2017.03.012.

Saludes, V., Folch, C., Antuori, A., González, N., García, D., Anoro, M.,... HepCdetect II Study Group. (2019). Utility of a one-step screening and diagnosis strategy for viremic HCV infection among people who inject drugs in Catalonia. The International Journal on Drug Policy, 74, 236-245. doi:10.1016/j.drugpo.2019.10.012.

Sánchez-Niubò, A., Sordo, L., Barrio, G., Indave, B. y Domingo-Salvany, A. (2020). Onset and progression of drug use in the general population of Catalonia, Spain. Adicciones, 32, 32-40. doi:10.20882/adicciones.1089.

Single, E., Collins, D., Easton, B, Harwood, H., Lapsley, H., Kopp, P.,... Wilson, E. (2003). International guidelines for estimating the costs of substance abuse. Geneva, Switzerland: World Health Organization (WHO). Recuperado de https://apps.who.int/iris/handle/10665/42603. 
UNODC (2017). World Drug Report 2017. Vienna, Austria: United Nations Office on Drugs and Crime (UNODC). Recuperado de https://www.unodc.org/wdr2017/index.html.

Vela, E., Clèries, M., Vella, VA., Adroher, C. y García-Altés, A. (2019). Análisis poblacional del gasto en servicios sanitarios en Cataluña (España): ¿Qué y quién consume más recursos? Gaceta Sanitaria, 33, 24-31. doi:10.1016/j. gaceta.2017.05.017.

Vella, V.A., García-Altés, A., Segura García, L., Ibáñez Martínez, N. y Colom Farran, J. (2018). Systematic review of guidelines in estimating social costs on drugs. Gaceta Sanitaria, 32, 481-487. doi:10.1016/j.gaceta.2017.10.009. 